\title{
Apport des Données Hydrodynamiques, Hydrochimiques et Isotopiques à l'amélioration des Connaissances des Aquifères du Parc du W du Niger, Région de Tillabéri
}

\author{
Ango Namata Habsatou, \\ Hassane Saley Abdel Kader, \\ Sandao Issouffou,
}

Département de Géologie, Faculté des Sciences et Techniques de l'Université Abdou Moumouni de Niamey, Niger

Lefebvre René,

Institut National de Recherche Scientifique du Québec, Canada

Ousmane Boureima,

Département de Géologie, Faculté des Sciences et Techniques de

l'Université Abdou Moumouni de Niamey, Niger

Doi:10.19044/esj.2020.v16n15p240 URL:http://dx.doi.org/10.19044/esj.2020.v16n15p240

\section{Résumé}

La zone d'étude, le Parc du W du Niger, est située dans la partie sudouest de la région de Tillabéri, département de Say, commune de Tamou, à $150 \mathrm{~km}$ au sud de Niamey, la capitale du Niger. Elle couvre une superficie de $2200 \mathrm{~km}^{2}$ et renferme environ $80 \%$ de la biodiversité du pays. Il a été reconnu, en 1996, comme réserve de la biosphère et Patrimoine mondial de l'UNESCO. Le Parc est traversé par la rivière Mékrou, affluent du fleuve Niger qui est à écoulement temporaire. Il comporte des forages destinés à soutenir l'étiage des mares artificielles au cours de la saison sèche, afin de limiter l'émigration des animaux sauvages vers les pays limitrophes (Bénin, Burkina Faso). L'objectif principal de cette étude est de contribuer à l'amélioration de la connaissance des aquifères du Parc du W du Niger, sollicités sur une période de l'année pour contribuer à la satisfaction des besoins en eau des animaux sauvages. La méthodologie appliquée est basée sur l'étude des coupes lithologiques des forages et des paramètres hydrodynamiques, ainsi que sur l'hydrochimie et la géochimie isotopique des eaux souterraines. Les résultats montrent que les débits maximum des forages varient de 0,85 à $10,50 \mathrm{~m}^{3} / \mathrm{h}$, avec une moyenne de $4,03 \mathrm{~m}^{3} / \mathrm{h}$ et un écart type de 3,84 . Les aquifères sont donc très peu productifs. Le suivi piézométrique a permis d'observer des hausses de niveaux statiques compris entre 1,61 et 4,09 $\mathrm{m}$, comparativement 
à ceux mesurés par le BRGM en 1990. Les valeurs de la transmissivité, comprises entre $7,1 \times 10^{-5}$ et $2,5 \times 10^{-4} \mathrm{~m}^{2} / \mathrm{s}$, sont relativement faibles et se situent dans la fourchette de celles trouvée en zone de socle du Niger et de la sous-région. Les eaux souterraines du Parc du W ont dans leur majorité des conductivités inférieures à $500 \mu \mathrm{S} / \mathrm{cm}$, à l'exception de celles prélevées dans les forages de Moussièmou et Yériyangou. La qualité chimique des eaux est relativement bonne, en dehors de celle de ces deux forages. Les eaux ont des faciès de type bicarbonaté calcique pour la plupart d'entre elles. Sur le diagramme de Schöeler Berkaloff, les eaux des forages de Moussièmou et Yériyangou sont représentées part des segments de droites parallèles, ce qui laisse penser que ces eaux ont la même origine. Par contre, pour les eaux des autres forages, les segments de droites s'entrecoupent, elles ont donc des origines différentes. Le temps de résidence des eaux souterraines déterminé à partir des âges non corrigés du carbone-14, varie de 3227 à 21641 ans. Ces résultats montent qu'il s'agit des eaux dont la composante actuelle est très faible, voire nulle. Ce sont par conséquent des eaux non renouvelables qui doivent être gérées avec précaution.

Mots-clés: Niger, Parc Du W, Eaux Souterraines, Hydrochimie, Géochimie Isotopique 


\title{
Contribution of Hydrodynamic, Hydrochemical and Isotopic Data to the Improvement of the Knowledge of the Aquifers of the W Park of Niger/Tillaberi Region
}

\author{
Ango Namata Habsatou, \\ Hassane Saley Abdel Kader, \\ Sandao Issouffou,
}

Département de Géologie, Faculté des Sciences et Techniques de

l'Université Abdou Moumouni de Niamey, Niger

Lefebvre Rene,

Institut National de Recherche Scientifique du Québec, Canada

\section{Ousmane Boureima,}

Département de Géologie, Faculté des Sciences et Techniques de

l’Université Abdou Moumouni de Niamey, Niger

\begin{abstract}
The study area, the W Park of Niger, is located in the south-western part of the Tillaberi region in the Say department. This department is a commune of Tamou, $150 \mathrm{~km}$ south of Niamey, which is the capital of Niger. It covers an area of $2,200 \mathrm{~km}^{2}$ and contains about $80 \%$ of the country's biodiversity. In 1996, it was recognized as a biosphere reserve and UNESCO World Heritage Site. The park is crossed by the Mekrou River, a tributary of the Niger River which is a temporary flow. It has boreholes to support the low water level of the artificial ponds during the dry season, so as to limit the emigration of wild animals to neighbouring countries (Benin, Burkina Faso). This paper aims to contribute to the improvement of the knowledge of the aquifers of the W Park of Niger, which has been used over a period of the year to meet the water needs of wild animals. The methodology applied is based on the study of borehole lithological sections and hydrodynamic parameters, as well as groundwater hydrochemistry and isotopic geochemistry. The results show that the maximum borehole flow rates vary from 0.85 to $10.50 \mathrm{~m}^{3} / \mathrm{h}$, with a mean of $4.03 \mathrm{~m}^{3} / \mathrm{h}$ and a standard deviation of 3.84 . The aquifers are therefore very unproductive. Piezometric monitoring has shown static level rises of between 1.61 and $4.09 \mathrm{~m}$ when compared to those measured by BRGM in 1990 . The transmissivity values ranging between $7.1 \times 10^{-5}$ and $2.5 \times 10^{-4} \mathrm{~m}^{2} / \mathrm{s}$ are relatively low and are within the range of those found in the basement zone of Niger and the sub-region. The majority of groundwater in the W Park has
\end{abstract}


conductivities of less than $500 \mu \mathrm{S} / \mathrm{cm}$. This is with the exception of that taken from the Moussièmou and Yériyangou boreholes. The chemical quality of the water is relatively good excluding that of these two boreholes. Most of the waters contain calcium bicarbonate facies. In Schöeler Berkaloff's diagram, the waters of the Moussièmou and Yériyangou boreholes are shown as segments of parallel lines, which suggests that these waters have the same origin. In the case of waters from other boreholes, the straight line segments intersect and, therefore, have different origins. The residence time of groundwater, determined from the uncorrected carbon-14 ages, varies from 3227 to 21641 years. These results show that these are the waters with very little or no current component. They are non-renewable waters that need to be carefully managed.

Keywords: Niger, W Park, Groundwater, Hydrochemistry, Isotopic Geochemistry

\section{Introduction}

Situé en Afrique de l'Ouest, dans la partie sud-ouest du Niger, la zone d'étude, le Parc national du W, couvre une superficie de $2200 \mathrm{Km}^{2}$ et se situe à $150 \mathrm{Km}$ de Niamey, la capitale du Niger. Ce Parc renferme une importante diversité biologique. Il est entouré d'une zone tampon constituée des réserves totale et partielle de Tamou $\left(74,445 \mathrm{~km}^{2}\right)$ et de Dosso $\left(3060 \mathrm{~km}^{2}\right)$ respectivement. Le Parc national du $\mathrm{W}$ fait partie du vaste complexe écologique dénommé WAP (W-Arly-Pendjari), qui s'étend sur $16000 \mathrm{~km}^{2}$, et est partagé entre trois pays, le Bénin, le Burkina Faso et le Niger (UICN, 2017).

Le Parc du W du Niger dispose d'un réseau hydrographique comprenant un petit tronçon du fleuve Niger, le seul cours d'eau permanent du Parc, mais aussi la Mékrou, affluent du Niger, et des "koris" à écoulement saisonnier (Figure 2). A ces cours d'eau s'ajoutent quelques mares naturelles et artificielles, plans d'eau constituant les principales sources d'abreuvement des animaux sauvages. Cependant, ces eaux superficielles sont relativement éloignées les unes des autres et s'assèchent dans leur majorité pendant la saison sèche (MESU/DD, 2015). D'où la nécessité de faire appel aux eaux souterraines pour les renforcer. En effet, il s'observe des mouvements des espèces fauniques vers la partie béninoise ou burkinabé du Parc du W pendant la période chaude, en quête d'eau. La présente étude a pour objectif de contribuer à remédier à cette situation en améliorant les connaissances sur les aquifères du Parc. 


\section{Contexte général de l'étude}

\subsection{Localisation}

Le Parc du "W "du Niger est situé dans le Sud-Ouest du Niger, à environ $150 \mathrm{~km}$ de Niamey, dans la commune rurale de Tamou, département de Say, Région de Tillabéri (Figure 1). Il s'étend entre $11^{\circ} 54^{\prime}$ et $12^{\circ} 35^{\prime}$ de latitude Nord, $02^{\circ} 04^{\prime}$ et $02^{\circ} 50^{\prime}$ de longitude Est. Il couvre une superficie de 2200 $\mathrm{km}^{2}$, dont $25 \mathrm{~km}^{2}$ de plan d'eau. Le Parc du W fait partie du vaste complexe écologique dénommé WAP (W-Arly-Pendjari), qui s'étend sur $16000 \mathrm{~km}^{2}$, et est partagé entre trois pays, le Bénin, le Burkina Faso et le Niger (UICN, 2017). Son nom découle de la forme en $W$ que décrit le tracé du fleuve Niger dans la partie nord du Parc. Ses limites naturelles sont le fleuve Niger à l'Est, le Burkina Faso à l'Ouest, la rivière Tapoa au Nord et la rivière Mékrou au Sud. Le Parc du W du Niger est classé comme réserve de biosphère et patrimoine mondial de l'UNESCO depuis 1996 (ECOPAS, 2005).

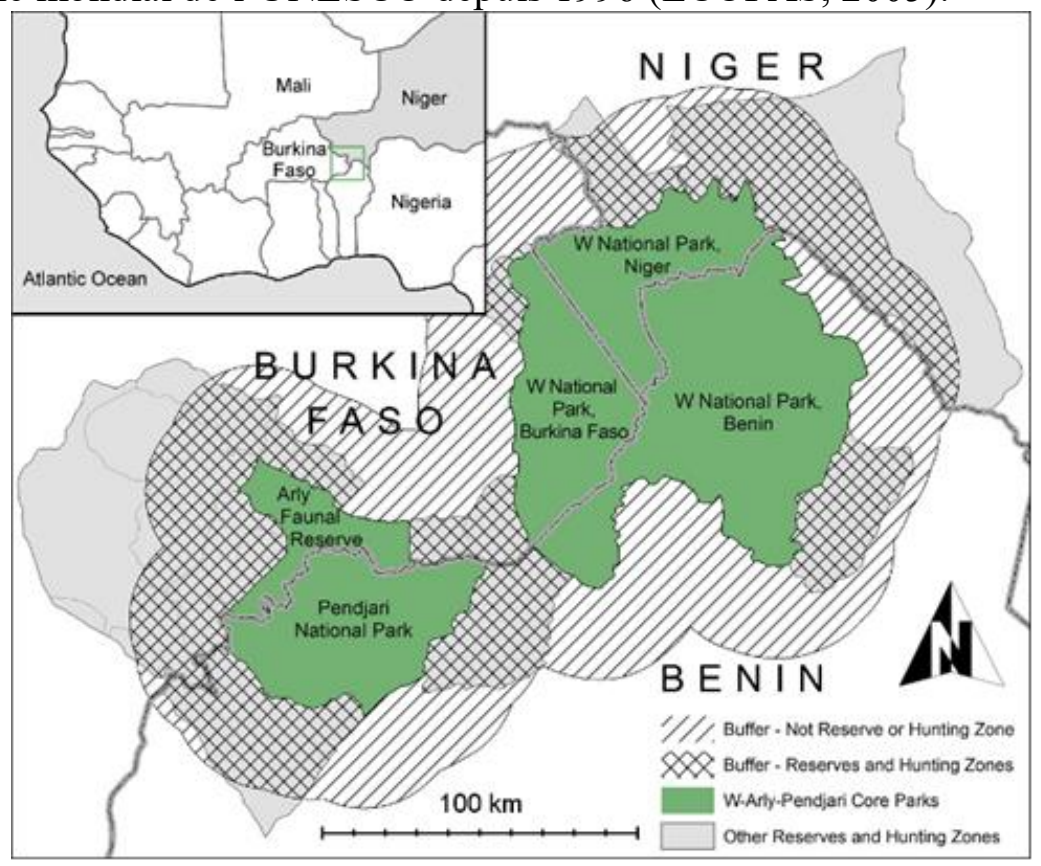

Figure 1. Localisation du complexe WAP (USGS, 2017 a)

\subsection{Cadre géomorphologique et hydrographique}

\subsubsection{Cadre géomorphologique}

La région du Parc du $\mathrm{W}$ du Niger est une vaste pénéplaine, caractéristique du vieux bouclier ouest africain qui affleure à l'extrémité nordest du bassin Voltaien. Son relief, très peu contrasté est, surtout, marqué par la chaine de l'Atakora et quelques escarpements (falaise de Gobnangou) (ECOPAS, 2005). La topographie à l'intérieur du Parc varie entre 150 et 300 $\mathrm{m}$ d'altitude (Figure 2). Le paysage est dominé par des plateaux tabulaires 
rocheux et cuirassés, découpés par des vallées parfois abruptes (Benoit, 1998). Le plateau principal du Parc s'élève à $300 \mathrm{~m}$, est entaillé par des cours d'eau saisonniers et est bordé par les vallées encaissées des cours d'eau relativement importants qui sont la Tapoa et la Mékrou.

\subsubsection{Cadre hydrographique}

Le réseau hydrographique est constitué de trois principaux cours d'eau (Figure 2) : le fleuve Niger, seul cours d'eau à écoulement permanent et ses deux affluents, la Mékrou et la Tapoa, à écoulement saisonnier. Il comprend également de petits cours d'eau (koris) à écoulements intermittents pendant la saison pluvieuse, et des mares naturelles ou artificielles qui sont semipermanentes (ECOPAS, 2002b).

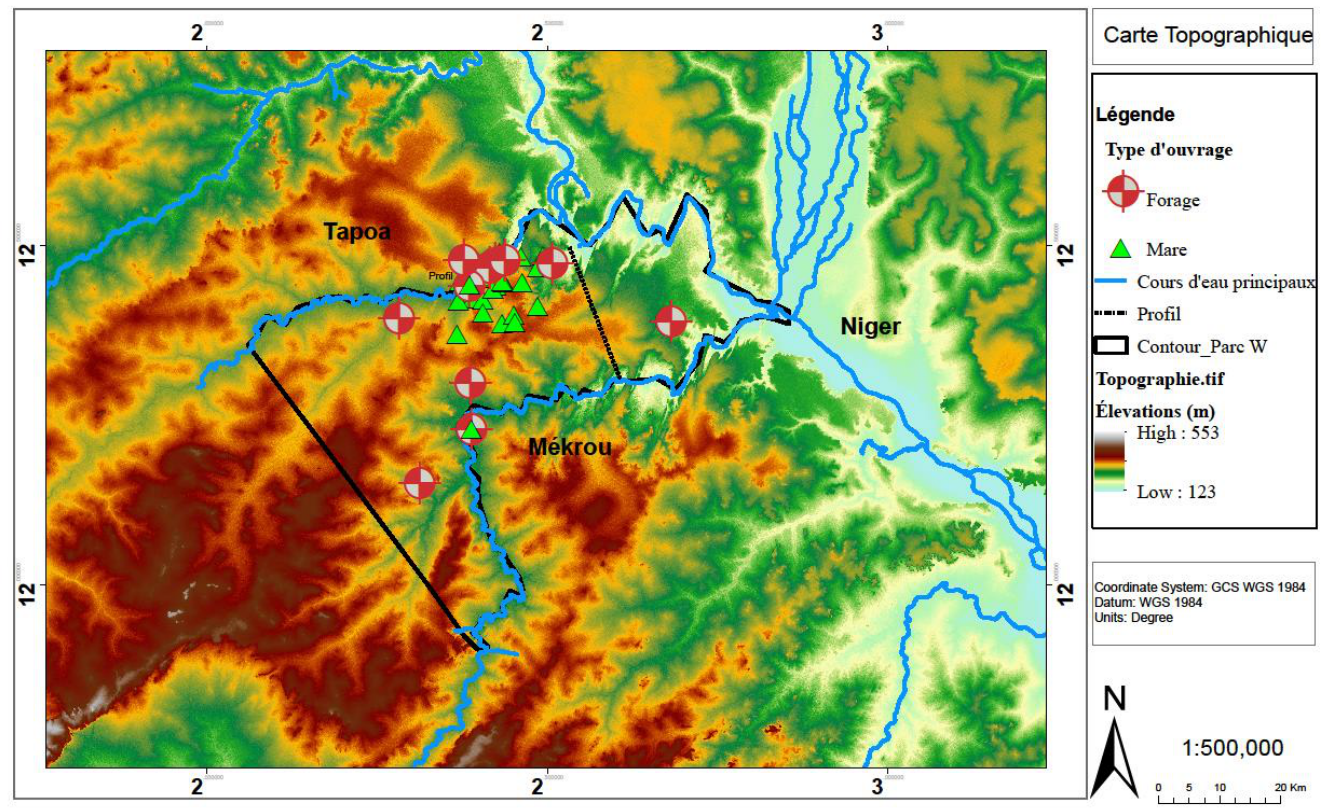

Figure 2. Topographie et cours d'eau principaux du Parc du W du Niger (Source des données de la NASA)

\subsection{Cadre climatique}

La zone du Parc national du "W" du Niger se situe en climat semi-aride de type sahélo-soudanien, avec des caractéristiques sahéliennes vers le nord et soudaniennes au sud. On distingue deux saisons au cours de l'année:

- Une saison sèche allant d'octobre à mi-mai ;

- Une saison pluvieuse de mi-mai à septembre.

La pluviométrie moyenne annuelle est de 600 à $700 \mathrm{~mm}$, vers le Nord du parc, et de 800 à $900 \mathrm{~mm}$ dans la partie sud. Ces pluies sont très variables 
dans l'espace et dans le temps (Kattiellou et al., 2014). Les précipitations maximales ont lieu au mois d'août (AQUASTAT / FAO, 2016).

La région du Parc du W est balayée par deux vents : l'harmatan chaud et sec, d'orientation nord-ouest et la mousson, vent humide de direction sudest venant de l'océan Atlantique, qui est à l'origine des précipitations (ArdoinBardin, 2004 ; Babayé, 2012). La valeur moyenne interannuelle de la température sur la période de 1994 à 2016 est de $28,6{ }^{\circ} \mathrm{C}$. La valeur maximale enregistrée en avril est de $40,1^{\circ} \mathrm{C}$, tandis que, la valeur minimale observée en janvier est de $18,5^{\circ} \mathrm{C}$ au niveau de la station synoptique de Fada N'Gourma. L'humidité relative varie de 22 à $84 \%$ sur la période allant de 2010 à 2016. Les faibles valeurs sont relevées pendant la saison sèche, tandis que les valeurs hautes sont enregistrées pendant la saison pluvieuse. L'évapotranspiration potentielle est de l'ordre de $2250 \mathrm{~mm} / \mathrm{an}$ dans le Parc du W (Ministère de l'hydraulique, 2005).

\subsection{Contexte géologique et hydrogéologique}

\subsubsection{Contexte géologique}

La géologie du Parc du W du Niger comprend surtout des formations de l'Infracambrien (grès voltaien), couvrant la majeure partie du Parc, mais aussi des formations du Précambrien moyen et supérieur, constituées de granito-gneiss (granites, gneiss etc.) et de lambeaux de séries volcanosédimentaires (schistes, micaschistes, amphibolites etc.). Enfin, la série du Continental Terminal repose, par endroits, en discordance sur l'Infracambrien (à l'Ouest et au Nord) (Rabeil, 2003), (Figure 3).

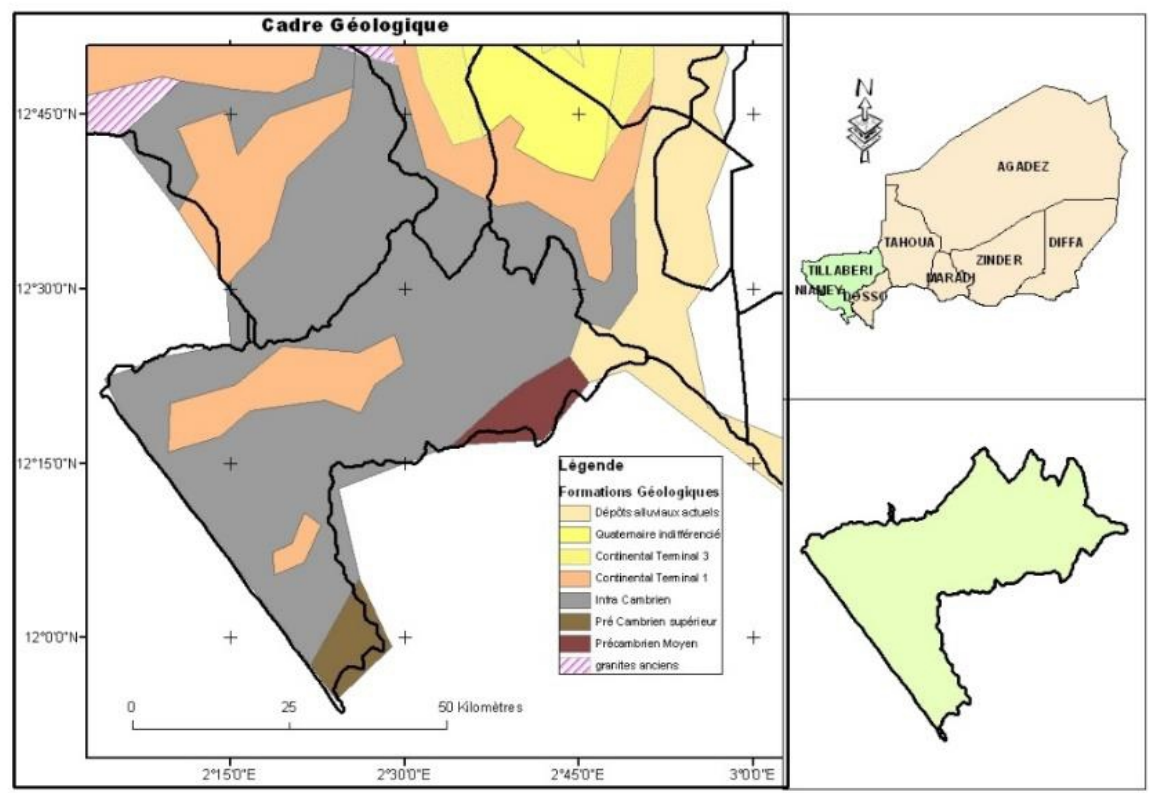

Figure 3. Géologie du Parc W du Niger (source, BRGM, 1961) 


\subsubsection{Contexte hydrogéologique}

Les formations géologiques du Parc du W renferment trois types d'aquifères $(\mathrm{MH} / \mathrm{E}, 2011)$ :

$\checkmark$ Les aquifères discontinus localisés dans les horizons altérés, fissurés et fracturés des grès de l'Infracambrien et du socle précambrien granito-gneissique ;

$\checkmark$ Les aquifères continus du Continental Terminal $\left(\mathrm{CT}_{3}\right)$;

$\checkmark$ Les aquifères alluviaux du Quaternaire en bordure du fleuve Niger et dans le fond de ses affluents.

Dans la zone du Parc, les forages existants captent essentiellement les aquifères du sédimentaire ancien (Grès du Voltaien) et du socle (granitogneiss du Précambrien) à des profondeurs variant de 50 à $91 \mathrm{~m}$. Leurs débits sont compris entre 0,85 et $10,50 \mathrm{~m}^{3} / \mathrm{h}$, tandis que les niveaux statiques se situent entre 1,10 et $49.24 \mathrm{~m}$ (BRGM, 1990), avec des venues d'eau observées entre 24 et $67 \mathrm{~m}$ de profondeur, ce qui montre que les eaux de ces aquifères circulent sous pression. Les valeurs de transmissivités varient de $7,1 \times 10^{-5}$ à $2,5 \times 10^{-4} \mathrm{~m}^{2} / \mathrm{s}$. Celles de la conductivité hydraulique, très faibles, sont comprises entre $2,4 \times 10^{-7}$ et $7,6 \times 10^{-6} \mathrm{~m} / \mathrm{s}$.

\section{Matériel et méthodes}

\subsection{Matériel}

Le matériel de cette étude est constitué par des outils de mesures de terrain, de prélèvement d'eau, de traitement de données et par les données elles-mêmes.

\subsubsection{Outils}

Les outils comprennent :

$\checkmark \quad$ Les équipements destinés aux essais de pompage (Compresseur à air, groupe électrogène, pompe hydraulique, appareils de mesure des volumes, Chronomètres, et autres accessoires) ;

$\checkmark$ Le petit équipement de terrain : GPS, appareil photo numérique, $\mathrm{pH}-$ mètre, conductivimètre, sonde de relevés de niveau d'eau, les bouteilles en polyéthylène pour le prélèvement des échantillons, une glacière pour la conservation des échantillons ;

$\checkmark$ Divers logiciels : Arcgis, Excel, OUAIP, Diagramme.

\subsubsection{Données}

Les principales données utilisées sont :

$\checkmark$ Les cartes topographique, géologique, climatique, hydrogéologique, sur la zone d'étude ; 
$\checkmark$ Les paramètres climatiques (hauteurs pluviométriques, température, humidité relative, évaporation et vent) des stations de Gaya et de Fada N'Gourma ;

$\checkmark$ Les rapports de thèses, de mémoires, des travaux divers en relation avec le thème de l'étude, et les articles scientifiques;

$\checkmark$ Les fiches techniques des forages d'eau (coupes lithologiques, données d'analyses physico-chimiques) issues des rapports de terrain ;

$\checkmark$ Les fiches de sondages miniers effectués dans le cadre de la prospection du phosphate ;

$\checkmark$ Les données acquises dans le cadre de la présente étude.

\subsection{Méthodes}

L'approche méthodologique adoptée porte sur l'acquisition des données et leur traitement:

\subsubsection{Acquisition des données}

Elle a porté sur les aspects suivants :

\subsubsection{Collecte et synthèse documentaires}

La collecte des données a été effectuée dans les centres documentaires des Ministères de l'Hydraulique et de l'Assainissement, et celui du Ministère de l'Environnement, ainsi qu'au niveau de l'Autorité du Bassin du Niger $(\mathrm{ABN})$ et des personnes ressources. Cette collecte a eu lieu aussi au niveau des sites Internet publics, et des institutions américaine et française dont, le National Océanic and Athmospheric Administration (NOAA) et le Système d'Informations Environnementales sur les Ressources en Eau et leur Mobilisation (SIEREM). La synthèse de ces documents collectés a permis d'améliorer les connaissances sur la thématique étudiée.

\subsubsection{Paramètres climatiques}

Les paramètres climatiques (hauteurs des pluies, température, humidité, évaporation et vents) des stations de Gaya et de Fada N'Gourma ont été obtenus au niveau des sites des institutions américaine et française, dont le National Océanic and Athmospheric Administration (NOAA) et le Système d'Informations Environnementales sur les Ressources en Eau et leur Mobilisation (SIEREM).

\subsubsection{Campagne d'échantillonnage des eaux destinées aux analyses physico-chimiques et isotopiques}

- Sélection des points d'eau

L'échantillonnage a porté sur cinq (5) des douze forages existants, accessibles et ayant fait l'objet de soufflage ou d'essai de pompage, deux 
mares et une eau de pluie. Aussi, il faut noter la disponibilité des données des essais de pompage de six (6) forages réalisés dans le Parc par le BRGM (1990).

\section{- Prélèvement}

Les bouteilles d'échantillonnage ont été préalablement étiquetées et marquées. Sur chaque étiquette figurent : le nom du site et celui du forage, la date, le type d'analyse à réaliser (les paramètres physico-chimiques et isotopiques), et le nom du conservateur. Cette étiquette est ensuite recouverte par un ruban d'adhésif, afin d'éviter que l'eau puisse l'altérer.

Après avoir observé toutes les précautions nécessaires afin d'éviter les contaminations extérieures, on procède au remplissage des bouteilles en rinçant chacune d'elle à plusieurs reprises, ainsi que, toutes les sondes des appareils. Ensuite, celles-ci sont remplies à l'aide d'une seringue en polyéthylène adaptable au filtre selon la quantité d'eau à prélever. Le volume d'eau filtré est de $30 \mathrm{~mL}$ pour les analyses chimiques et les isotopes stables, de $500 \mathrm{~mL}$ et $1000 \mathrm{~mL}$ respectivement pour le tritium $\left({ }^{3} \mathrm{H}\right)$ et le carbone $\left({ }^{14} \mathrm{C}\right)$. Pour ce dernier le conservateur utilisé est la soude caustique, $(\mathrm{NaOH}, 0,5 \mathrm{~mL}$ d'une solution $5 \mathrm{~N}$ ), il est introduit à l'aide d'une pipette de $1 \mathrm{~mL}$. Ainsi un total de 38 bouteilles ont été remplies. Par ailleurs, il est à noter que des mesures spécifiques, visant à éviter au maximum les échanges atmosphériques, ont été observées lors du prélèvement des échantillons destinés à l'analyse isotopique $\left({ }^{3} \mathrm{H}\right.$ et $\left.{ }^{14} \mathrm{C}\right)$. Ces échantillons ont été conservés et transportés dans une glacière jusqu'aux laboratoires.

\subsubsection{Techniques analytiques}

- Paramètres physiques $\left(\mathbf{p H}, \mathbf{C}, \mathbf{t}^{\circ}\right)$

Ils ont été mesurés in situ à l'aide d'un $\mathrm{pH}$-mètre (Unités $\mathrm{pH}$ ) de marque HANNA et un conductivimètre de marque WTW, LF92 équipé d'une sonde de température (température et conductivité) (Figure 4).

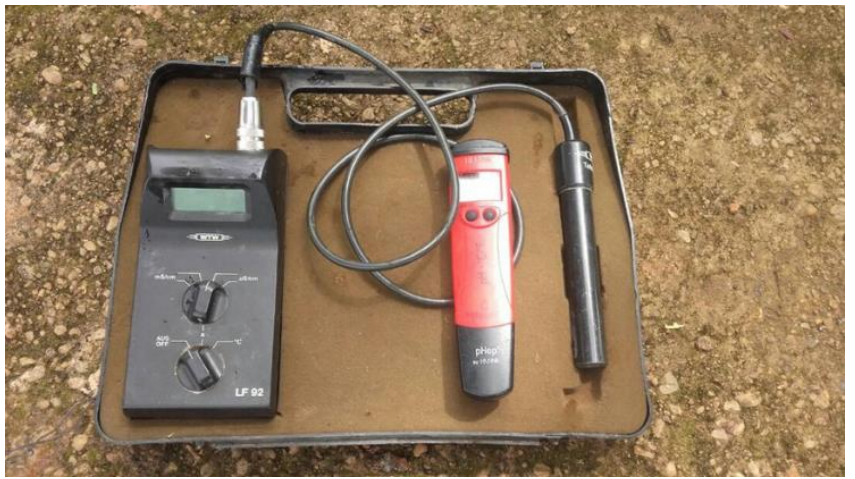

Figure 4. Photo du matériel de mesures physico-chimiques in situ 


\section{- Paramètres chimiques}

\section{- Analyse des anions}

Les cations ont été analysés par spectroscopie d'émission par plasma à couplage inductif (ICP) au laboratoire de l'Institut National de Recherche Scientifique du Québec (INRS). Les échantillons, préalablement filtrés, acidifiés et dilués au besoin, sont transformés en aérosol dans un nébuliseur puis injecté dans la source ICP. Cette dernière soumet les atomes constitutifs de l'aérosol à de de hautes températures de l'ordre de 6000 à $8000^{\circ} \mathrm{K}$. L'excitation fournie par l'ICP permet de détecter de nombreux cations en tenant compte des interférences potentielles. Les limites de détection pour les cations se situent entre 0,00009 et 0,009 .

\section{- Analyse des anions}

L'analyse des anions est effectuée par chromatographie ionique au laboratoire de l'Institut National de Recherche Scientifique du Québec (INRS). Cette méthode analytique d'échange d'ion est basée sur la séparation des molécules selon leurs charges. Il se produit des réactions ioniques entres les ions de charges opposées fixés sur la phase stationnaire entrainant ainsi leur rétention. Les limites de détection des anions varient de 0,004 à 0,005.

\section{- Paramètres isotopiques}

○ Isotopes stables $\left({ }^{18} \mathrm{O}\right.$ et $\left.{ }^{2} \mathrm{H}\right)$

- Détermination de ${ }^{18} \mathrm{O}$

L'oxygène $\left({ }^{18} \mathrm{O}\right)$ est déterminé par équilibre $\mathrm{H}_{2} \mathrm{O}-\mathrm{CO}_{2}$ en utilisant des procédures standard basées sur les principes d'Epstein et Mayeda (1953). La précision est de $\pm 0.2 \%$.

- Détermination de ${ }^{2} \mathrm{H}$

Le dosage du deutérium $\left({ }^{2} \mathrm{H}\right)$ est effectué sur l'hydrogène gazeux produit à partir d'eau réduite par le chrome chaud, selon les procédures décrites par Drimmie et al (2001a). La précision de cette technique est de $\pm 0,8 \%$.

Les rapports ${ }^{2} \mathrm{H} /{ }^{1} \mathrm{H},{ }^{17} \mathrm{O} /{ }^{18} \mathrm{O}$ et ${ }^{18} \mathrm{O} /{ }^{16} \mathrm{O}$ des échantillons d'eau à faible TDS sont mesurés à l'aide d'un appareil, Los Gatos Research (LGR) et Liquid Water Isotope Analyser (LWIA), modèle T-LWIA-45-EP, avec des précisions, $\delta^{2} \mathrm{H}$ $= \pm 0,8 \%$ et $\delta^{18} \mathrm{O}= \pm 0,2 \%$.

Les résultats sont exprimés en $\delta \%$ vs VSMOW (Vienna Standard Mean Ocean Water) et en VSLAP (Vienna Standard Light Antarctic Precipitation). Isotopes radioactifs

- Détermination de ${ }^{3} \mathrm{H}$

La technique utilisée pour doser le tritium est la spectrométrie de scintillation liquide (LSC).

La limite inférieure de détection a été arbitrairement fixée à 6 et l'écart-type (sd) à 8 . 
○ Détermination $\mathrm{du}^{14} \mathrm{C}$

Les échantillons sont convertis en dioxyde de carbone pur qui est piégé dans des tubes de verre Pyrex de $6 \mathrm{~mm}$ de diamètre extérieur, puis ils sont ensuite analysés par spectrométrie de masse avec accélérateur pour la détermination $\mathrm{du}{ }^{14} \mathrm{C}$.

Il est à noter que toutes les analyses isotopiques $\left({ }^{18} \mathrm{O},{ }^{2} \mathrm{H},{ }^{3} \mathrm{H}\right.$ et $\left.{ }^{14} \mathrm{C}\right)$ ont été effectuées au laboratoire de l'Université de Waterloo (Canada).

\subsubsection{Détermination des paramètres hydrodynamiques}

- Relevé des niveaux statiques

Dans le cadre de ce travail, l'état des ouvrages (absence de dispositif permettant de mesurer le niveau, pose des pompes sur certains ouvrages, etc), n'a pas permis d'effectuer un suivi de la fluctuation des niveaux statiques des forages. Néanmoins, il existe quelques données de relevé de niveau d'eau des ouvrages réalisés par le BRGM en 1990 qui ont été utilisées.

- Les débits des forages

La détermination des caractéristiques des forages (débits instantané, débit optimal, type d'écoulement, etc.) a été effectuée par des essais de pompage par palier de courte durée dans le cadre de cette étude. Ainsi, trois paliers sans interruption, d'une durée d'une heure de temps chacun, ont été réalisés, le tout suivi d'une remontée. Les débits de ses paliers sont respectivement Q1 (estimé à partir du développement à la pompe), Q2=2 Q1 et $\mathrm{Q} 3=3 \mathrm{Q} 1$. Pendant ces essais, les niveaux dynamiques sont mesurés périodiquement avant, pendant et après les essais de pompage ainsi que les temps.

\section{- Les valeurs de la transmissivité}

En dehors des données relatives aux valeurs de transmissivité existantes, qui vont être utilisées, il a été réalisé dans le cadre de cette étude, des essais de pompage de longues durées sur trois forages qui sont ceux de Moussièmou, Bata et Tapoa Barrage. Ces pompages ont duré de 6 à 12 heures avec des débits constants de $1,45 \mathrm{~m}^{3} / \mathrm{h}$ pour le forage de Moussièmou, de 10,6 $\mathrm{m}^{3} / \mathrm{h}$ pour Bata et de $4,2 \mathrm{~m}^{3} / \mathrm{h}$ pour Tapoa Barrage. Par ailleurs, ces essais ont été suivis de l'observation des remontées de niveaux respectives pendant $6 \mathrm{~h}$ $30 \mathrm{mn}$ pour Moussièmou, $6 \mathrm{~h}$ de temps pour Bata et $7 \mathrm{~h}$ de temps pour Tapoa Barrage.

\subsubsection{Traitement des données}

\subsubsection{Détermination des moyennes statistiques des facteurs climatiques}

Les moyennes interannuelles des précipitations, de l'humidité, de la température et celles de l'évapotranspiration potentielle et réelle, calculées par le logiciel WHAT 4.2.0-Beta2 sont représentées sur un graphique. 


\subsubsection{Paramètres hydrodynamiques \\ - Variabilité des niveaux statiques}

Les données disponibles sur les niveaux statiques sont celles du rapport du BRGM (1990) et les mesures de niveaux effectuées dans le cadre de ce travail. Ces données vont être superposées à un modèle conceptuel afin de déterminer l'évolution du comportement de la nappe. Le programme de suivi de ces niveaux d'eau, initié dans le cadre de l'étude, n'a malheureusement pas été opérationnel, car il n'existe pas de piézomètres dans la zone du Parc du W.

- Détermination des débits des forages et de la transmissivité des nappes

- Les débits des forages

Il s'agit de déterminer le débit critique des forages et le rabattement maximal à partir des courbes établies selon la formule $s / Q=f(Q)$ et $s=f(Q)$. Par ailleurs, on déterminera les paramètres $\mathrm{B}$ et $\mathrm{C}$ de l'équation de Jacob $\mathrm{s}=$ $\mathrm{BQ}+\mathrm{CQ}^{2}$ qui exprime respectivement la charge linéaire et quadratique

\section{- Détermination de la transmissivité}

Les données issues des essais de pompage peuvent être traitées à l'aide du logiciel OUAIP du BRGM (2015 b) disponible en ligne ou par la méthode graphique de Jacob.

$\checkmark$ Détermination des valeurs de la transmissivité à l'aide du logiciel OUAIP : elle consiste à introduire les valeurs des rabattements et des temps de pompage, connaissant le débit de pompage. Les graphiques et les valeurs de transmissivité, de conductivité hydraulique et le coefficient d'emmagasinement sont automatiquement générés ;

$\checkmark$ Détermination des valeurs de la transmissivité par la méthode graphique de Jacob.

En utilisant l'équation de Jacob (1946), le rabattement

$s=\frac{2,3}{4 \pi} \times \frac{\mathrm{Q}}{\mathrm{T}} \log \left(\frac{2,25 \times \mathrm{T} \times \mathrm{t}}{r^{2} \mathrm{~S}}\right)$, on trace la courbe $\mathrm{s}=\mathrm{f}(\log (\mathrm{t}))$. On prolonge cette droite jusqu'à l'axe des abscisses pour lire la valeur de $t_{0}$. Ensuite, on calcule la pente de la droite $(\Delta \mathrm{s})$. On calcule la valeur de la transmissivité dans un rayon de $\log$ de 10 à partir de la formule $: T=\frac{2,30 \times Q}{4 \pi \Delta s}$ avec $Q$ représentant le débit de pompage.

\subsubsection{Paramètres physico-chimiques}

\section{- Paramètres physiques}

La comparaison des valeurs de la conductivité, du $\mathrm{pH}$ et de la température des eaux de la zone à celles des paramètres physiques (conductivité, $\mathrm{pH}$ et température) permet de déterminer le degré de salinité des eaux de la zone, leur acidité, leur basicité, ainsi que l'origine de la température. Les valeurs 
moyennes maximales de ces paramètres physiques ont été comparées également aux normes de potabilité des eaux de l'OMS, 2011.

- Paramètres chimiques

- Balance ionique

Pour vérifier la fiabilité des résultats analytiques, on détermine la balance ionique qui est donnée par l'expression suivante :

$$
B I=\frac{\sum\left(\text { cations }-\sum \text { anions }\right)}{\sum \text { cations }+\sum \text { anions }} \times 100
$$

Si cette valeur de la balance est inférieure ou égale à 6\%, les analyses sont jugées acceptables.

Il est à noter que les cations et les anions sont en meq/L.

- Représentation graphique des éléments chimiques

Les représentations graphiques couramment utilisées sont le diagramme de Piper et celui de Schöeler Berkaloff.

Le Diagramme de Piper représenté par deux triangles séparés par un losange permet la classification des eaux en faciès chimiques, tandis que, celui de Schöeler Berkaloff représenté par des droites verticales reliées par des segments de droites permet de déterminer l'origine des eaux selon que les segments de droites sont parallèles ou s'entrecoupent.

- Aptitude des eaux à la consommation humaine

Les concentrations minimales et maximales des ions majeurs constituées par des anions $\left(\mathrm{Cl}, \mathrm{HCO}_{3}{ }^{-}, \mathrm{SO}_{4}, \mathrm{NO}_{3}\right)$ et des cations $(\mathrm{Mg}, \mathrm{Na}, \mathrm{Ca}, \mathrm{K})$, les valeurs moyennes des paramètres physiques (température, $\mathrm{pH}$ et conductivité), ainsi que, les teneurs des éléments mineurs $\left(\mathrm{Fe}, \mathrm{F}, \mathrm{NO}_{2}\right)$ et en traces ( $\mathrm{Al}, \mathrm{As}, \mathrm{B}, \mathrm{Ba}$, $\mathrm{Cd}, \mathrm{Co}, \mathrm{Cr}, \mathrm{Cu}, \mathrm{Mn}, \mathrm{Mo}, \mathrm{Ni}, \mathrm{P}, \mathrm{Pb}, \mathrm{Br}, \mathrm{S}, \mathrm{Sb}, \mathrm{Sc}, \mathrm{Se}, \mathrm{Si}, \mathrm{Sn}, \mathrm{Sr}, \mathrm{Ti}, \mathrm{V}, \mathrm{Zn}$ ) sont comparées aux normes de potabilité de l'OMS.

\subsubsection{Paramètres isotopiques}

Les isotopes stables de la molécule d'eau $\left({ }^{18} \mathrm{O}\right.$ et $\left.{ }^{2} \mathrm{H}\right)$ seront portés sur un graphique représentant les droites météorique mondiale et locale permettant de déterminer le mécanisme de recharge et l'origine des eaux en les comparants aux moyennes pondérées des précipitations.

Les âges $\mathrm{du}{ }^{14} \mathrm{C}$, non corrigés, sont utilisés pour donner une indication sur le temps de résidence de l'eau souterraine. Un modèle numérique élaboré à partir de la solution de Chesnaux et al. (2005) permet de confirmer ce temps de résidence de l'eau dans l'aquifère. 
III. Résultats et Discussions

3.1 Évolution des paramètres climatiques et détermination du bilan hydrique

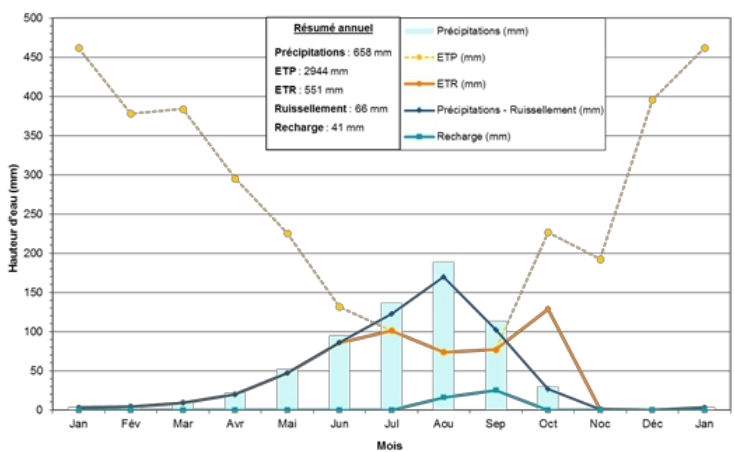

Figure 5. Graphique montrant l'évolution des différents paramètres climatiques et le taux de recharge minimal pour la période 1994-2016

Le graphique (Figure 5) représentant les principaux paramètres climatiques (pluviométriques, température, évapotranspiration potentielle et réelle) montrent que l'évapotranspiration potentielle est supérieure de plusieurs ordres de grandeurs aux précipitations, en dehors du mois d'août où elle devient inférieure aux pluies. Ainsi, on observe au mois d'août, une recharge probable qui varie de 41 à $77 \mathrm{~mm}$ en fonction des hauteurs de pluies qui sont respectivement de 668 et de $709 \mathrm{~mm}$.

\subsection{Caractéristiques hydrodynamiques}

\subsubsection{Niveaux statiques}

Les niveaux statiques des forages du Parc du W du Niger mesurés en 1990 (BRGM, 1990) se situent entre 1,10 et $49,24 \mathrm{~m}$ et sont approximativement les mêmes que ceux mesurés dans les ouvrages de ce Parc dans le cadre du présent travail. Il est à noter que des valeurs identiques ont été observées dans la zone périphérique du parc, c'est-à-dire le secteur de Tamou. En considérant que les niveaux d'arrivées d'eau se situent entre 24 et $67 \mathrm{~m}$, il y'a lieu d'admettre que les nappes sont sous pression, justifiant ainsi les niveaux statiques observés. Par ailleurs, des variations interannuelles et/ou saisonnières des niveaux statiques ont été observées dans les ouvrages. Cette variation est de l'ordre de 3,67 m dans plusieurs ouvrages, se traduisant ainsi par des remontées comprises entre 1,91 et 3,67 m, ce qui pourrait s'expliquer par une recharge des nappes ou leur développement, intervenu après leur mise en exploitation. 


\subsubsection{Détermination des débits des forages, des transmissivités et conductivités hydrauliques des aquifères}

\subsubsection{Débits des forages}

L'application du logiciel OUAIP du BRGM (2015b) aux données obtenues au cours des essais par paliers de débits de courte durée, a permis de déterminer les débits critiques des forages, leurs rabattements maxima et leurs pertes de charges linéaires et quadratiques (Figure 6a, b, c et Tableau 1). On constate alors que la productivité diffère selon les forages. Lorsque le rabattement est fort, le forage tant à être dénoyé.

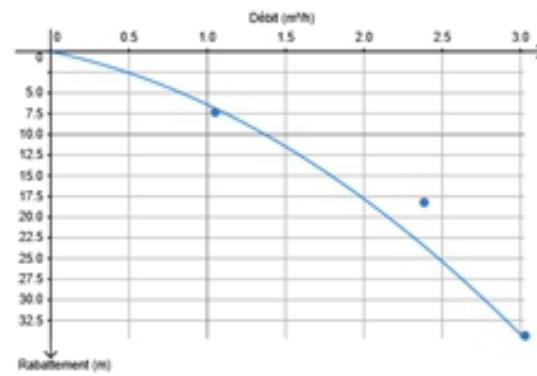

a)

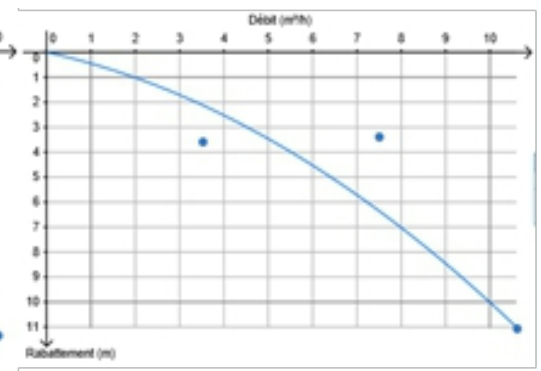

b) Forage Tapoa Bata

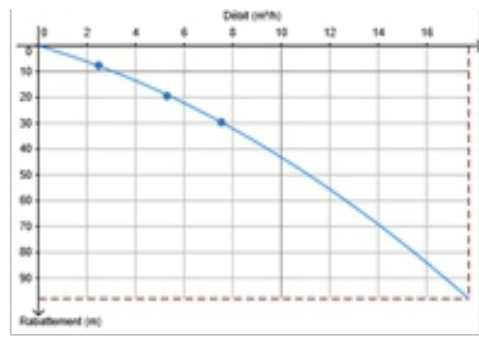

c) Forage Tapoa Barrage Figure 6. Courbes caractéristiques des forages du Parc du W du Niger

Tableau 1. Caractéristiques des forages du Parc du W, déterminés par les essais par pallier.

\begin{tabular}{|l|c|c|c|c|c|}
\hline \multicolumn{7}{|c|}{ Caractéristique de quelques des forages du Parc W } \\
\hline Nom des Forages & $\begin{array}{c}\text { Débit critique } \\
(\mathrm{m} 3 / \mathrm{h})\end{array}$ & $\begin{array}{c}\text { Rabattement } \\
\text { maximale }(\mathrm{m})\end{array}$ & $\begin{array}{c}\text { Débit spécifique } \\
(\mathrm{m} / \mathrm{h} / \mathrm{m})\end{array}$ & $\begin{array}{c}\text { Coefficient. B } \\
(\mathrm{h} / \mathrm{m})\end{array}$ & $\begin{array}{c}\text { Coefficient.C } \\
(\mathrm{h} 2 / \mathrm{m} 5)\end{array}$ \\
\hline Tapoa Moussiemou & 1.56 & 12.2 & 0.128 & 3.89 & 2.5 \\
\hline Tapoa Bata & 6.7 & 4.77 & 1.40 & 0.402 & 0.06 \\
\hline Tapoa Barrage & 4.5 & 15.4 & 0.29 & 2.76 & 0.156 \\
\hline
\end{tabular}

\subsubsection{Transmissivités et conductivités hydrauliques des aquifères}

Les résultats des essais $(\mathrm{s}=\mathrm{f}(\mathrm{t}))$ de longue durée traités par le logiciel OUAIP du BRGM (2015b) ont été utilisés pour établir les graphiques de la figure (Figure 7) ayant permis de déterminer les valeurs de transmissivité, de conductivité hydraulique et de coefficient d'emmagasinement (Tableau 2) de l'aquifère étudié. On constate que la transmissivité obtenue avec le forage de Bata est plus élevée que celles des forages de Moussièmou et Tapoa Barrage. En comparaison avec les résultats antérieurs, les valeurs de transmissivité obtenues dans le cadre de cette étude sont du même ordre de grandeur que ceux obtenus par le BRGM (1990). On peut en déduire que les caractéristiques des aquifères n'ont pas varié entre 1990 et 2018 (Tableau 2). Ce qui implique que les réserves des aquifères sont restés peu ou pas exploitées. 


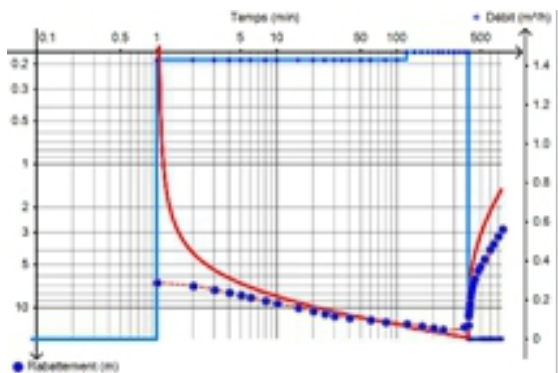

a) Moussièmou

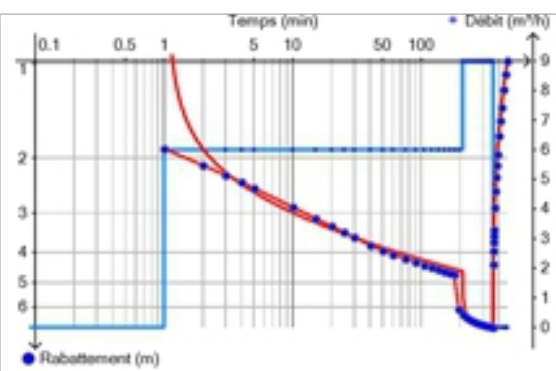

b) Tapoa Bata

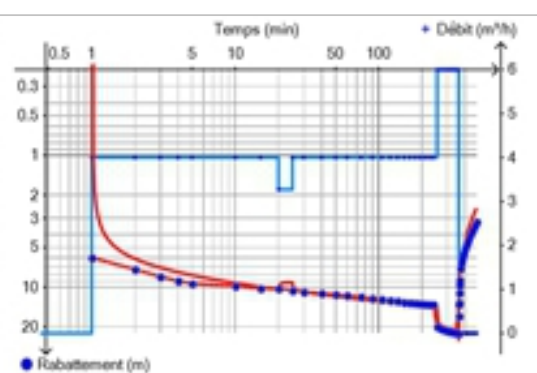

c) Tapoa Barrage

Figure 7. Graphiques montrant les courbes de descente et de remontée des forages du Parc du W (la courbe en bleu représente la courbe expérimentale et en rouge, la courbe théorique)

Tableau 2. Comparaison des caractéristiques hydrodynamiques des forages du Parc W du Niger obtenues en 2018 avec ceux du BRGM (1990), pour des essais réalisés sur 3 forages

Caractéristiques hydrodynamiques des forages du Parc W du Niger

\begin{tabular}{|l|c|c|c|c|c|c|c|c|}
\hline & \multicolumn{2}{|c|}{$\mathbf{T}\left(\mathbf{m}^{2} / \mathbf{s}\right)$} & \multicolumn{2}{c|}{$\mathbf{K}(\mathbf{m} / \mathbf{s})$} & \multicolumn{2}{c|}{ Coef. S } & \multicolumn{2}{c}{ Coef. NASH } \\
\cline { 2 - 10 } Nom des ouvrages & $1990^{1}$ & $2018^{2}$ & $1990^{1}$ & $2018^{2}$ & $1990^{1}$ & $2018^{2}$ & $1990^{1}$ & $2018^{2}$ \\
\hline Tapoa Barrage & $6.02 \mathrm{E}-05$ & $7.12 \mathrm{E}-05$ & $2.86 \mathrm{E}-06$ & $3.39 \mathrm{E}-06$ & $3.61 \mathrm{E}-02$ & $1.16 \mathrm{E}-03$ & 0.890 & 0.949 \\
\hline Tapoa Moussiemou & $2.26 \mathrm{E}-05$ & $1.55 \mathrm{E}-05$ & $3.43 \mathrm{E}-07$ & $2.36 \mathrm{E}-07$ & $2.52 \mathrm{E}-01$ & $1.08 \mathrm{E}-01$ & 0.935 & 0.765 \\
\hline Tapoa Bata & $2.58 \mathrm{E}-04$ & $2.49 \mathrm{E}-04$ & $7.17 \mathrm{E}-06$ & $6.92 \mathrm{E}-06$ & $2.59 \mathrm{E}-01$ & $1.19 \mathrm{E}-02$ & 0.911 & 0.935 \\
\hline
\end{tabular}

(1) : Données du BRGM ;

(2) : Données issues de ce travail.

\subsection{Caractéristiques physico-chimiques des eaux}

\subsubsection{Paramètres physiques des eaux}

Les valeurs des conductivités électriques obtenues sont comprises entre 278 et $2420 \square \mathrm{S} / \mathrm{cm}$, avec une moyenne de 937,8 et un écart type de 60,39 . Ainsi, on constate une minéralisation très différente entre les eaux des différents forages. Ce qui pourrait traduire que cette minéralisation s'est acquise par contacte entre l'eau et les roches et/ou par pollution. Par ailleurs, ces minéralisations sont très différentes de celles des eaux de précipitation $(15 \square \mathrm{S} / \mathrm{cm}$ ) et des eaux de surface (19 à $60 \square \mathrm{S} / \mathrm{cm}$ ). Les $\mathrm{pH}$ des eaux, varient de 6,4 à 8,6, indiquant des eaux légèrement acides à basiques. Quant aux valeurs des températures mesurées, elles varient entre 25 et $32 \square \mathrm{C}$, ce qui reflète la température moyenne de l'air atmosphérique.

\subsubsection{Paramètres chimiques des eaux}

\subsubsection{Balance ionique}

La balance ionique de la quasi-totalité des échantillons est inférieure à $6 \%$. Néanmoins, les eaux faiblement chargées (l'eau de surface, l'eau de 
pluie et l'eau du forage de Bata) ont des balances ioniques variant de 7, 18 et $20 \%$, celles-ci pourraient s'expliquer par des erreurs de manipulations compte tenu des faibles teneurs en ions des échantillons. Ainsi, les balances ioniques de l'ensemble des analyses des eaux souterraines sont aptes à des interprétations chimiques.

\subsubsection{Faciès chimiques}

La composition géochimique est très variable dans les eaux souterraines du Parc W du Niger. On constate qu'il y a trois types d'eau : les eaux ayant un faciès du type bicarbonaté calcique (c'est le cas de la plupart d'entre elles), celles ayant un faciès du type bicarbonaté calcique et magnésien, et enfin, celles ayant un faciès du type chloruré sulfaté calcique et magnésien (Figure 8).

Le diagramme de Schöeler Berkaloff montre que les forages de Moussièmou et Yériyangou présentent des segments de droites parallèles, ce qui pourrait traduire le fait que ces eaux ont la même origine. Par contre, pour les eaux des autres forages, les segments de droite s'entrecoupent, indiquant que ces eaux sont éventuellement d'origines différentes (Figure 9).

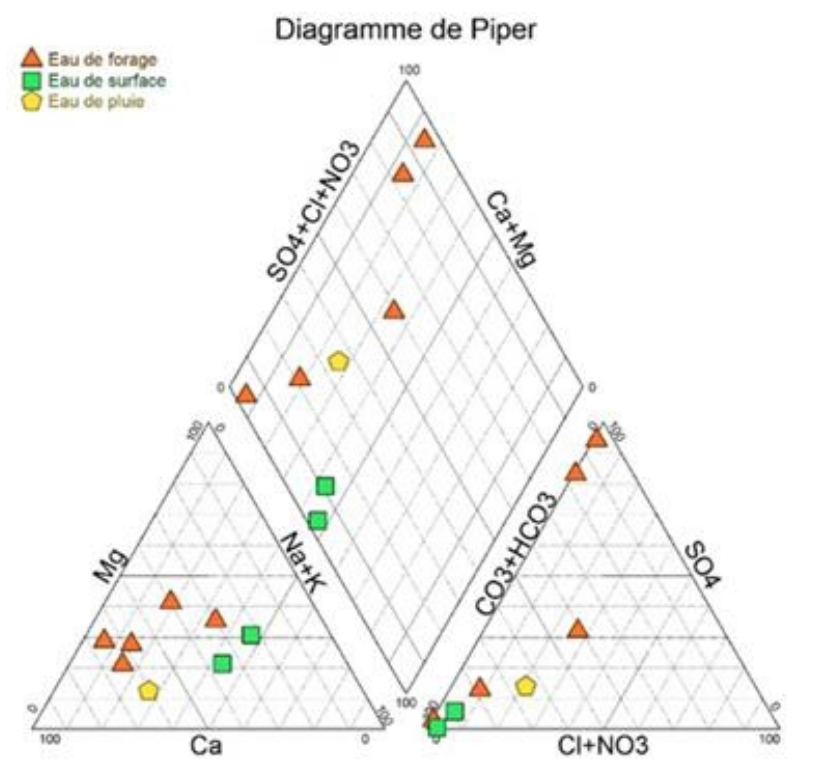

Figure 8. Positions des échantillons d'eau sur le diagramme de Piper. 


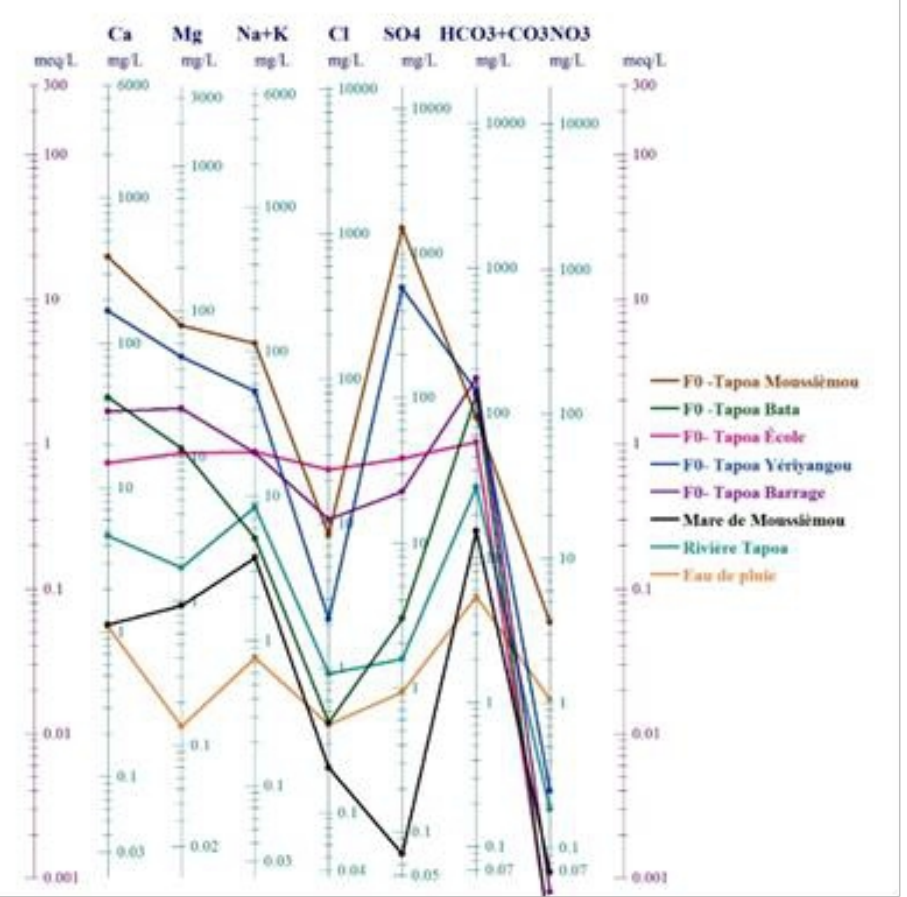

Figure 9. Présentation graphique de la composition des échantillons d'eau sur le diagramme de Schöeler-Berkaloff

\subsubsection{Aptitude des eaux à la consommation humaine}

La qualité chimique des eaux du Parc du W est dans l'ensemble bonne pour la consommation humaine. Néanmoins, le forage de Moussièmou et Yériyangou présentent des concentrations élevées en calcium et sulfates, supérieures à la norme requise par l'OMS (2011). Le forage de Moussièmou présente également un dépassement de la teneur en magnésium. Par ailleurs, les valeurs des TDS de ces forages sont très élevées. Les conductivités sont inférieures à la norme OMS, $2011(500 \mu \mathrm{S} / \mathrm{cm})$ pour l'ensemble des échantillons à l'exception des eaux de ces deux forages. Des méthodes d'adoucissement des eaux souterraines telles que, la précipitation à la chaux éteinte ou la coagulation floculations par le sulfate d'aluminium pourraient être envisagées.

\subsection{Caractéristiques isotopiques des eaux du Parc du $W$ du Niger}

\subsubsection{Isotopes stables}

Le report des teneurs en isotopes stables (18O) des eaux sur les droites météoriques mondiale et locale (Figure 10) permet de décrire les différentes catégories d'eau analysées: 
- $\quad$ l'eau de pluie analysée est très enrichie en isotopes stables (180). Elle est collectée en fin de saison de pluie, ce qui pourrait être à la base de cet enrichissement. Cette eau ne semble pas être à l'origine des eaux trouvées dans la rivière et dans la mare qui sont issues des eaux de précipitations moins évaporées (de l'époque actuelle).

les eaux souterraines, qui ont des teneurs en isotopes stable (180) variant de - 4,8 à - $4 \%$, pourraient avoir une composante plus ou moins importante des eaux de précipitations actuelles. En effet, les moyennes pondérées des pluies actuelles sont de - 2 à - $4 \%$ vs VSMOW. Par contre, les forages dont les eaux ont des teneurs isotopiques variant de - 6 à - 5,5\%o sont des eaux anciennes, dont la composante des pluies actuelles est très faible, voire nulle.

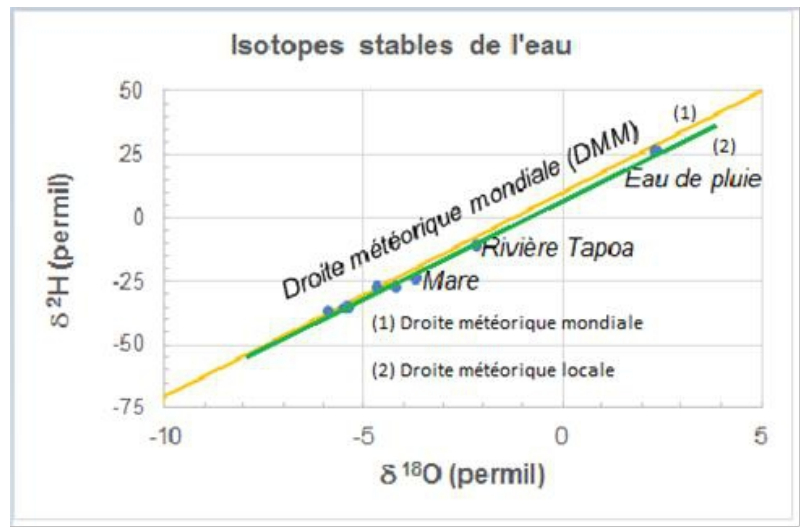

Figure 10. Projection des eaux échantillonnées en 2018 dans le Parc W du Niger sur le diagramme $\delta 18 \mathrm{O}$ vs $\delta 2 \mathrm{H}$ (Craig ,1961)

\subsubsection{Isotopes radioactifs}

La variation des âges carbone-14 non corrigés (Tableau 3) entre 21641 et 3227 ans confirme le résultat tiré des données de l'oxygène-18, à savoir que les eaux souterraines étudiées pourraient recevoir actuellement ou à une période humide du Quaternaire, un apport limité d'eaux pluviales. En effet, si ces âges non corrigés étaient compris entre 21641 et $18117 \mathrm{ans}$, il s'agirait d'eaux souterraines très anciennes.

Tableau 3. Valeurs des isotopes stables et du carbone-14 des eaux du Parc du W

\begin{tabular}{|c|c|c|c|c|c|c|c|c|c|c|}
\hline Échantillons & $\delta 180$ & Result & Repeat & $82 \mathrm{H}$ & Result & Repeat & $14 \mathrm{C}$ & & RCAge & \\
\hline & $\mathrm{H} 2 \mathrm{O}$ & vSMOW & $0.2 \% 0$ & $\mathrm{H} 2 \mathrm{O}$ & VSMOW & $\pm 0.8 \%$ & $\mathrm{pmC}$ & 10 error & $B P$ & 10 error \\
\hline Forse Tapos Mous siemou & $x$ & -4.20 & -4.12 & $\mathrm{x}$ & -27.21 & -26.38 & 18. 181 & 0.1 & 13703 & 44 \\
\hline F o sge de lécole & $\mathrm{x}$ & -5.39 & & $\mathrm{x}$ & -35.03 & & 15.844 & 0.14 & 14800 & 71 \\
\hline F $\propto$ sge Tapos B sta & $\mathrm{x}$ & -4.67 & -4.65 & $\mathrm{x}$ & -26.88 & -26.74 & 68.92 & 0.22 & 3227 & 26 \\
\hline Mare de Mouss iemou & $\mathrm{x}$ & -3.67 & & $\mathrm{x}$ & -23.96 & & 91.747 & 0.289 & 692 & 25 \\
\hline Forsge de Yériyangou & $\mathrm{x}$ & -5.89 & & $\mathrm{x}$ & -36.44 & & 10.484 & 0.087 & 18117 & 67 \\
\hline F $\propto$ rge Tapos Barrage & $\mathrm{x}$ & -5.47 & -5.45 & $\mathrm{x}$ & -35.15 & -34.23 & 6.781 & 0.074 & 21841 & 88 \\
\hline Rivière Tapos & $\mathrm{x}$ & -2.14 & & $\mathrm{x}$ & -11.12 & & 101.825 & 0.271 & Modern & \\
\hline Eau de pluie & $\mathrm{x}$ & 2.33 & 2.32 & $\mathrm{x}$ & 26.33 & 25.62 & & & & \\
\hline
\end{tabular}




\section{Conclusion}

Cette étude montre que les aquifères du Parc du W du Niger ont des potentialités hydriques relativement limitées, à l'exception de l'aquifère capté par le forage de Bata où le débit du forage peut atteindre $10 \mathrm{~m} 3 / \mathrm{h}$, avec un débit spécifique de $1,40 \mathrm{~m} 3 / \mathrm{h} / \mathrm{m}$.

Les eaux souterraines semblent être relativement anciennes avec des âges de l'ordre de 20000 ans. Par conséquent, en attendant des études hydrogéologiques beaucoup plus avancées, il semble que la contribution des eaux souterraines à l'approvisionnement en eau des animaux serait limitée. Aussi, il souhaitable, dans l'état actuel des connaissances, d'augmenter le nombre de points d'eau de surface et de surcreuser les mares afin de les rendre permanentes et d'éviter ainsi les déplacements des animaux de la partie nigérienne du parc vers les pays frontaliers.

Enfin, les études hydrogéologiques, hydrodynamiques et hydrogéochimiques du Parc du W demandent à être approfondies, en vue d'améliorer les connaissances sur les aquifères en présence, afin de déterminer leur contribution éventuelle à l'alimentation en eau des animaux sauvages.

\section{References:}

1. Abdou Babayé, M. S. (2012). Evaluation des ressources en eau dans le bassin de Dargol (Liptako-Niger), Thèse de doctorat. Université de Liège (Belgique) et Université Abdou Moumouni (Niger), 236 p.

2. Ardoin_Bardin, S. (2004). Variabilité hydro-climatique et impacts sur le ressources en eau de grands bassin hydrographiques en zone Soudano-Sahélienne, Thèse de doctorat de l'Université de Montpelier II, $437 \mathrm{p}$.

3. AQUASTAT/FAO (2016). Système d'information sur l'eau et l'agriculture, base des données pour l'Organisation des Nations Unies pour l'alimentation et l'agriculture.

4. Benoit, M. (1998). Statut et usages du sol en périphérie du Parc national $\mathrm{du} \mathrm{W}$ du Niger, Tome 1 , Contribution à l'étude du milieu naturel et des ressources végétales du canton de Tamou et du Parc du $<<\mathrm{W}>>$. ORSTOM, représentation au Niger. Paris, Niamey.

5. BRGM (1990). Fiches des ouvrages, Projet Autorité du Liptako gourma (ALG).Ministère de l'hydraulique et de l'assainissement, Niamey/ Niger

6. BRGM (2015b). OUAIP: outil d'aide à l'interprétation des pompages d'essais. https://www.brgm. fr/production-scientifique/logicielsscientifiques/ouaip-outil-aide-interpretation-pompages-essais [accédé en juin 2019]

7. Kattiellou, G, L., Maurizio, B. \& Moussa, M. (2014). Caractérisation climatique de la région de Tillabéri. Rapport $\mathrm{N} \square$ 1, Niamey/ Niger, 34 
8. MESU/DD (2015). Projet de réhabilitation des points d'eau dans la Reserve de Biosphère de $\mathrm{W}$ du Niger. Ministère de l'Environnement de la Salubrité Urbaine et du Développement Durable, Niamey / Niger $18 \mathrm{p}$

9. Ministère de l'Hydraulique et de l'Environnement (2011). Gestion intégrée et durable des ressources en eau en Afrique axée sur les États membres de la région du sahel : Etat de la connaissance et la gestion des eaux souterraines au Niger. Projet AIEA RAF 7011. Vienne, $49 \mathrm{p}$

10. Ousmane, B. (1988). Étude géochimique et isotopique des aquifères du socle de la bande du sahélienne du Niger (Liptako, sud-Maradi et Zinder Est). Thèse Univ. Niamey, 175p

11. Programme Régional Parc W / ECOPAS (2005). Plan d'aménagement et de gestion de la Reserve de Biosphère Transfrontalière W-20062010, Volume 1 : Etat des lieux.

12. Ouagadougou, $228 \mathrm{p}$.

13. Rabeil, T. (2003). Distribution potentielle des grands mammifères dans le Parc du $\mathrm{W}$ au Niger, Thèse Univ. Paris VII, 451 p., https://tel.archives-ouvertes.fr/tel-00006931.

14. UICN (2017). COMPLEXE W-ARLY-PENDJARI (extension du W Niger), rapport d'évaluation. Niamey, Niger. 17p.

15. USGS (2017a). Case study. The W-Arly-Pendjari Transboundary Biosphere Reserve. In West Africa land use cover dynamics, https//eros.usgs.gov/westafrica/case study/ W-arly-pendjari transboundary-biosphere-reserve.

16. USGS (2017b). Climate in West Africa: Land use and land cover dynamics, https// eros.usgs.gov/ Westafrica/ node/ 157. 